\title{
IMAGEM CORPORAL DE MILITARES: UM ESTUDO DE REVISÃO
}

\author{
MS. JAIRO JOSÉ MONTEIRO MORGADO \\ Mestre em Educação Física pela Universidade Federal de Juiz de Fora (UFJF), Pesquisador do Instituto \\ de Pesquisa da Capacitação Física do Exército (IPCFEx) (Rio de Janeiro - Rio de Janeiro - Brasil) \\ E-mail: jairomorgado@bol.com.br
}

\section{DRA. FABIANE FROTA DA ROCHA MORGADO}

Mestre em Educação Física pela Universidade Federal de Juiz de Fora (UFJF),

Doutora em Educação Física pela Universidade Estadual de Campinas

(UNICAMP) (Campinas - São Paulo - Brasil)

E-mail: fabi.frm@hotmail.com

\section{DRA. MARIA DA CONSOLAÇÃO GOMES CUNHA FERNANDES TAVARES}

Doutora em Medicina Interna pela Universidade Estadual de Campinas (UNICAMP), Professora Titular da Faculdade de Educação Física da Universidade Estadual de Campinas (FEF/ UNICAMP) (Campinas - São Paulo - Brasil)

E-mail:mcons@fef.unicamp.br

\section{DRA. MARIA ELISA CAPUTO FERREIRA}

Pós-Doutora em Educação pela Universidade do Estado de São Paulo (USP), Professora Adjunta da Faculdade de Educação Física e Desportos da Universidade

Federal de Juiz de Fora (FAEFID/UFJF) (Juiz de Fora - Minas Gerais - Brasil)

E-mail: caputoferreira@terra.com.br

\section{RESUMO}

A imagem corporal é a representação mental da identidade corporal, o que torna importante seu estudo para a individualização das ações educativas, pedagógicas e de atividades físicas em diferentes grupos de indivíduos, inclusive entre militares. Este estudo objetivou realizar uma revisão de literatura sobre a imagem corporal de homens militares e verificar a relação desta com a prática de atividade física no contexto das Instituições Militares. Foram rastreados estudos nas bases de dados Scopus, SportDiscus, Scielo, Web of Science e PsycINFO por meio de descritores de busca referentes à imagem corporal, militar e atividade física. De um total de 10865 artigos encontrados, somente nove abordavam o tema imagem corporal de homens militares. Portanto, há uma carência de estudos nesta área, o que torna necessário a realização de pesquisas futuras sobre a imagem corporal de militares, visando trazer beneficios para este grupo.

PALAVRAS-CHAVE: Militares; imagem corporal; insatisfação corporal; atividade física. 
O Tema imagem corporal tem sido estudado por diversas áreas do conhecimento, quais sejam: Educação Física, Sociologia, Psicologia, Psiquiatria, entre outras. O foco de estudos tem sido diferentes grupos de indivíduos, especialmente crianças, jovens, idosos e pessoas com transtornos alimentares. Entretanto, a abordagem do público militar parece ser escassa.

A imagem corporal pode ser entendida como uma complexa associação de informações referentes à identidade corporal. É uma estrutura singular e indivisível, que permite ao indivíduo se reconhecer como a mesma pessoa a todo instante, estando em permanente processo de construção e reconstrução. É a imagem mental da identidade corporal, que por sua vez, diz respeito às experiências e vivências, as quais trazem ao sujeito, em todo momento, novas e diferenciadas sensações e percepções. Os aspectos sociais, emocionais e fisiológicos se inter-relacionam, interferindo na remodelação constante da imagem corporal (SCHILDER, 1980; TAVARES, 2003).

As primeiras pesquisas sobre imagem corporal com o público masculino sugeriam que os homens possuíam percepções positivas a respeito do próprio corpo, sobretudo, a respeito do peso e gordura corporal. Os homens, normalmente, possuíam pequenos índices de transtorno alimentar, altos níveis de satisfação com o corpo e menores índices de insatisfação com seu sobrepeso. Neste contexto, muitos pesquisadores concluíam que o público masculino era considerado livre de preocupações a respeito da imagem corporal, ao contrário do que era observado em mulheres e garotas (MCCREARY, 20 I I).

A partir da última década, as pesquisas realizadas com homens tomaram um novo enfoque. A preocupação destas não era mais em relação ao peso e gordura corporal. Descobriu-se que fazia mais sentido focar sobre massa muscular e sua distribuição no corpo, visto que muitos garotos e homens desejavam uma "busca pela musculosidade" (do original: "drive for muscularity"). Pessoas com níveis mais altos de busca pela musculosidade possuíam maiores riscos de abusar de anabolizantes e outros suplementos que prometiam ganho de massa muscular. Desde então, as pesquisas revelaram que este grupo também está sujeito a alterações de imagem corporal e o número de estudos nesta área cresceu significativamente nos últimos anos (McCREARY, 20 I I).

Nos estudos da imagem corporal, pode-se conceber que a atividade física tem um efeito positivo na imagem corporal. Schilder (1980) preconiza que as sensações provenientes do interior do corpo possuem significado e estão conectadas na estrutura da imagem corporal, influenciando as ações motoras. Logo, o 
movimento é um facilitador do desenvolvimento saudável da Imagem Corporal. Tavares (2003) afirma que a atividade física pode fazer parte da construção da identidade corporal, proporcionando ao indivíduo vivências que o possibilite ser consciente de seus aspectos afetivos, sociais e fisiológicos. Ginis e Bassett (201 I), por sua vez, destacam que a imagem corporal é positivamente impactada pela atividade física.

No Exército Brasileiro (EB), é comum a prática de atividades físicas tradicionais e militares. Estas podem ser realizadas tanto por intermédio de treinamento físico quanto por atividades diversas, tais como: bivaques, ' pistas de aplicações militares, ${ }^{2}$ marchas a pé, ${ }^{3}$ acampamentos, ${ }^{4}$ entre outras, que possuem espaço primordial na rotina da vida militar (BRASIL, 20 I 0). O significado das atividades físicas desenvolvidas com o público militar do EB, da Marinha e da Aeronáutica pode ter impactos positivos ou negativos na vida de cada indivíduo.

De um lado, as atividades físicas podem proporcionar impactos positivos e saudáveis na imagem corporal destes sujeitos. Estas atividades visam habilitar o indivíduo para o cumprimento de missões de combate, as quais objetivam proporcionar-Ihe rusticidade e resistência, qualidades que the possibilita "durar na ação" ${ }^{5}$ em situações de desgaste e de estresse (BRASIL, 2008). Nesse sentido, os militares têm a possibilidade de explorar e ampliar suas próprias capacidades, por meio de novas vivências e experiências corporais diversificadas. Em consequência, podem ampliar o conhecimento de seu corpo, estruturando sua identidade corporal.

Por outro lado, as atividades físicas podem estar relacionadas a atributos negativos sobre a imagem corporal dos homens militares. Os sujeitos que ingressam nas Instituições Militares trazem sua história de vida, suas experiências, sensações e percepções, sua individualidade e são apresentados a um sem número de novas experiências e sensações, onde a individualidade passa a ser confrontada com os interesses da coletividade. Nesse contexto, a identidade corporal do indivíduo, matriz básica da imagem corporal, se depara com a procura pela integralização de uma "identidade militar". Esta identidade militar é uma expressão bastante enfatizada no contexto da vida militar e se refere à rigidez de atitudes, pensamentos e comportamentos relacionados ao próprio corpo. Ela é forjada pelo desenvolvimento de atributos, atitudes e valores necessários ao profissional militar.

\footnotetext{
I. Acampamentos temporários ao ar livre, normalmente realizado apenas com o material levado pelo militar em sua mochila.

2. Pistas nas quais os indivíduos buscam transpor uma série de obstáculos, naturais ou artificiais, como rios, matas densas, muros, cercas, etc.

3. Caminhadas forçadas realizadas com fardamento de combate, com o armamento e equipamento individuais, como a mochila.

4. Jornadas de trabalho em período integral, nas quais são realizadas atividades de simulação de combate, durante vários dias, em ambiente rural, com a utilização de barracas e similares para pernoite.

5. Permanecer com capacidade para combater.
} 
Frente ao exposto, a compreensão das relações existentes entre a imagem corporal de homens militares e a prática de atividade física no contexto das instituições militares tem relevância na definição de estratégias para formação de militares competentes e saudáveis. Entretanto, será que as atividades físicas propostas aos homens militares facilitam o desenvolvimento de sua imagem corporal? Será que as relações existentes entre a prática de atividade física e a imagem corporal dos militares são suficientemente estudadas?

Este estudo objetiva realizar uma revisão da literatura sobre imagem corporal de homens militares e verificar a relação desta com a prática de atividade física no contexto das Instituições Militares.

\section{MATERIAIS E MÉTODOS}

TIPO DE PESQUISA

Esta pesquisa é uma revisão de literatura que tem por objetivo coletar material a ser analisado e avaliado sobre imagem corporal de homens militares.

\section{BASE DE DADOS}

Foram utilizadas cinco bases de dados: Scopus, SportDiscus, Scielo, Web of Science e PsycINFO. As bases foram escolhidas devido à variedade de temas que englobam e pela vasta gama de publicações que versam sobre imagem corporal presentes em seus bancos de dados.

Para realização das pesquisas foram combinadas as seguintes palavras:

- Military + Exercise;

- Military + Physical activity;

- Military + Physical exercise;

- Military + Fitness;

- Military + Exercise + "Body Image";

- Military + Physical activity + "Body Image";

- Military + Physical exercise + "Body Image";

- Military + Fitness + "Body Image"; e

- Military + "Body Image". 


\section{CRITÉRIOS DE INCLUSÃO E EXCLUSÃO}

Foram incluídos os artigos que atendessem às seguintes exigências: os artigos deveriam ter sido publicados até Agosto de 201 I e deveriam conter a população militar do sexo masculino. O critério de inclusão que aborda somente o público masculino se justifica porque este grupo de pessoas ainda é majoritário nas Instituições Militares e corresponde a aproximadamente $96 \%$, em média, do efetivo profissional. ${ }^{6}$

Foram excluídos os artigos que não tivessem como foco a população militar e que não tivessem como objeto de estudo a imagem corporal.

\section{PROCEDIMENTOS}

Inicialmente, foi feita a determinação do tema, que se deu através da experiência do primeiro autor com as atividades militares e pelo interesse no tema imagem corporal, além da impressão de que os estudos nesta área não são muito explorados nas Forças Armadas. Em seguida, foi feita uma busca de artigos nas bases de dados (realizada através do Portal Capes). Houve, então, uma seleção prévia dos artigos encontrados, por meio da análise e seleção dos artigos que realmente tratavam o tema a ser estudado. Por fim, foram aplicados os critérios de inclusão e exclusão, visando selecionar apenas os artigos de interesse para este estudo.

\section{RESULTADOS E DISCUSSÕES}

Nas cinco bases de dados, utilizando as nove combinações, foi encontrado um total de 10865 artigos, como exemplificado na Tabela I.

6. O efetivo de homens contabilizados na Marinha da Brasil corresponde a 55.223 de um total de 58.663 militares, ou seja, 94,13\% dos militares são homens (Disponível em: http://www.mar.mil.br/imprensa/arquivos_PDF/ carta_mmb2.pdf, acesso em março de 20।0).

O efetivo de homens contabilizados no Exército Brasileiro corresponde a 197.822 de um total de 202.995 militares, ou seja, 97,45\% dos militares são homens (Disponível em: http://www.fab.mil.br/portal/capa/index. php?page =imprensa, acesso em março de 20 I0).

$\mathrm{O}$ efetivo de homens contabilizados na Força Aérea Brasileira corresponde a 55.761 de um total de 60.935 militares, ou seja, 91,51\% dos militares são homens (Disponível em:, http://www.defesabr.com/eb.htm\#Atuais, acesso em março de 20।0.) 
Tabela I. Resultado da pesquisa nas bases de dados

\begin{tabular}{lllllll}
\hline & \multicolumn{2}{l}{ BASE DE DADOS } & & & \\
DESCRITORES & SCOPUS & SPORTDISCUS & SCIELO & $\begin{array}{l}\text { WEB OF } \\
\text { SCIENCE }\end{array}$ & PSYCINFO \\
\hline Military + Exercise & 3459 & 534 & 22 & 1130 & 437 \\
Military + Physical Activity & 1065 & 115 & 2 & 217 & 78 \\
Military + Physical Exercise & 963 & 30 & 2 & 35 & 20 & \\
Military + Fitness & 1342 & 550 & 6 & 428 & 316 \\
Military + Exercise + "Body Image" & 11 & 2 & 0 & 0 & 2 & \\
Military + Physical Activity + "Body Image" & 7 & 0 & 0 & 1 & 0 & \\
Military + Physical Exercise + "Body Image" & 6 & 0 & 0 & 0 & & 0 \\
Military + Fitness + "Body Image" & 8 & 0 & 0 & 0 & 1 & \\
Military + "Body Image" & 38 & 5 & 0 & 5 & 28 & \\
TOTAL & 6899 & 1236 & 32 & 1816 & 882 & \\
TOTAL GERAL DAS BASES & 10865 & & & & & \\
\hline
\end{tabular}

Fonte: $O$ autor (201 I)

Em uma análise prévia dos totais de publicações de cada base de dados (Scopus, Sportdiscus, Scielo, Web of Science e PsycINFO), chama atenção o acentuado número de estudos com a população militar relacionados, sobretudo, aos quatro primeiros descritores, os quais podem sintetizar a área do Treinamento Físico: Exercise, Physical Activity, Fitness, Physical Exercise. Este fato possibilita refletir sobre o enfoque proeminente que o treinamento físico possui no contexto militar.

Desde tempos mais antigos, a história da Educação Física retrata a busca do preparo físico pelos guerreiros da antiguidade, pelos egípcios em 4.000 antes de Cristo, chineses em 3.000 antes de Cristo, por cretenses, gregos, e vários outros povos e culturas. Além destes, outros grupos responsáveis pela defesa e realização de interesses comuns, da mesma forma que as Instituições Militares, preocupavam-se com o desempenho físico de seus membros, visando à preparação para o combate, para a caça, pesca e defesa de interesses comuns (GUTIERREZ, 1980). Uma outra reflexão que vincula o treinamento físico e o público militar está baseada na Educação Física militarista, bastante utilizada até bem pouco tempo no Brasil. Seu objetivo principal era a obtenção, por meio de variados treinamentos físicos, de uma juventude capaz de suportar o combate, a luta, a guerra (GHIRALDELLI, 1988). Estes fatores associados podem justificar o número significativo de estudos com militares na área do Treinamento Físico.

Dando continuidade à análise da tabela I, pode-se constatar que, quando o descritor de busca "Body image" é associado aos descritores relacionados ao Treinamento Físico, ou ainda, quando este descritor é associado com a palavra "military", 
o número de publicações é reduzido de forma brusca. Sabe-se que a maioria dos militares do Brasil são homens. A limitação de estudos com a população militar masculina contrapõe-se às sugestões de alguns pesquisadores, como Grammas e Schwartz (2009), que enfatizam a necessidade de realização de pesquisas de imagem corporal com homens, tendo em vista a importância de aumentar o conhecimento nesta área, sobretudo aquele relacionado à avaliação da imagem corporal, com medidas validadas e apropriadas para esta população. Os autores sugerem ainda estudos longitudinais e investigações qualitativas com o público masculino.

Outros autores também enfatizam a importância de estudar a imagem corporal do público masculino, tendo em vista que as dimensões de imagem corporal do homem, assim como das mulheres, são suscetíveis a transtornos (GINIS et al., 2005; SWAMl et al., 2008). Ginis et al. (2005) constataram alterações em aspectos da imagem corporal de homens após a realização de treinamentos de força, confirmando que este público é suscetível de alteração em sua imagem corporal. Cafri e Thompson (2004) e Sabino (2007) afirmam que os comportamentos relacionados à busca pela musculosidade podem estar associados a alterações na imagem corporal e podem resultar em efeitos negativos, físicos e psicológicos, para a saúde, como o uso de anabolizantes e outros hormônios.

$\mathrm{Na}$ Tabela 2, apresentada a seguir, chama atenção que, no Scielo, uma importante base de dados que abrange estudos de países ibero-americanos, não foram encontradas pesquisas quando o descritor "military" foi cruzado com o termo "Body image". No Brasil também não foi encontrado este tipo de estudo, o que revela um quadro de carência de pesquisas nesta área. Turtelli, Tavares e Duarte (2002) reportam que estudos sobre imagem corporal abordam diferentes grupos de investigação, como, por exemplo, praticantes de atividade física e sedentários, mulheres atletas e não atletas, estudantes de ensino médio, bailarinas e não bailarinas, crianças do sexo masculino e feminino, modelos e prostitutas, negros e brancos. Pode-se perceber que o público militar não tem sido o foco de pesquisadores nesta área.

É importante destacar que a lacuna de estudos com homens militares brasileiros pode estar vinculada à escassez de opções metodológicas válidas e precisas para avaliar a imagem corporal destas pessoas. Campana e Tavares (2009) afirmam que não há disponível, no contexto nacional, um instrumento apropriado para medir a imagem corporal do público masculino e revelam a importância de adaptar ou criar novas medidas para esta finalidade. 
Tabela 2. valores percentuais de publicações em cada base de dados analisada.

\begin{tabular}{lllcccc}
\hline \multirow{2}{*}{$\begin{array}{l}\text { DESCRITORES } \\
\text { PRINCIPAIS }\end{array}$} & $\begin{array}{c}\text { DESCRITORES } \\
\text { SECUNDÁRIOS }\end{array}$ & $\begin{array}{c}\text { Valores percentuais de estudos encontrados em cada base de dados } \\
\text { SCOPUS }\end{array}$ & $\begin{array}{l}\text { SPORTDISCUS } \\
\text { SCIELO }\end{array}$ & $\begin{array}{c}\text { WEB OF } \\
\text { SCIENCE }\end{array}$ & PSYCINFO \\
\hline Military & $\begin{array}{l}\text { Exercise; physical acti- } \\
\text { vity; Physical exercise } \\
\text { e Fitness }\end{array}$ & $98,98 \%$ & $99,43 \%$ & $100 \%$ & $99,67 \%$ & $96,48 \%$ \\
$\begin{array}{l}\text { Military e"Body } \\
\text { Image" }\end{array}$ & $\begin{array}{l}\text { Exercise; Physical acti- } \\
\text { vity; Physical exercise } \\
\text { e Fitness }\end{array}$ & $0,46 \%$ & $0,16 \%$ & $0 \%$ & $0,05 \%$ & $0,34 \%$ \\
$\begin{array}{l}\text { Military e "Body } \\
\text { Sem outros descri- } \\
\text { tores }\end{array}$ & $0,55 \%$ & $0,40 \%$ & $0 \%$ & $0,27 \%$ & $3,17 \%$ \\
TOTAL & $100 \%$ & $100 \%$ & $100 \%$ & $100 \%$ & $100 \%$ \\
\hline
\end{tabular}

Fonte: $\mathrm{O}$ autor (201 I)

Cumpre esclarecer que alguns artigos apareceram de forma repetida nas diversas pesquisas realizadas nos bancos de dados. Entretanto, após a triagem inicial para eliminação das duplicações e dos artigos que não versavam sobre o tema, foram aplicados os critérios de inclusão e exclusão, os quais mostraram a existência de apenas nove artigos que exploraram o tema da imagem corporal em homens militares. Os estudos sobre militar e imagem corporal foram reunidos em quatro grupos: o primeiro deles é relacionado ao tabagismo e à obesidade; o segundo é referente a transtornos alimentares, o terceiro refere-se à insatisfação corporal e o quarto engloba um estudo que investiga "membro fantasma".

Com relação ao primeiro grupo, três estudos foram realizados na área de tabagismo e obesidade (JAMES et al., 1997; WARD et al., 2003; DOBMEYER et al., 2005). No estudo realizado por James et al. (1997), foi proposto um programa de intervenção ao sobrepeso para militares ativos do Exército Americano. $\bigcirc$ programa era composto por um período de internação intensiva de 3 semanas e visava a perda de peso dos militares. Ele teve seguimento com serviços ambulatoriais, com palestras sobre estilos de vida saudáveis, exercícios físicos, nutrição, controle de emoções e atitudes. Durante o programa, uma equipe de psicólogos avaliava o desenvolvimento da imagem corporal dos militares. Vale lembrar que, no período da realização deste estudo, a preocupação dos pesquisadores a respeito dos homens estava voltada para investigações acerca do peso e percentual de gordura corporal.

Ward et al. (2003) realizaram um estudo com militares da Força Aérea Americana para verificar possíveis ligações entre tabagismo, atividade física e controle de peso. Militares fumantes foram recrutados para este estudo. Dentre estes, foi constatado que aqueles mais ativos demonstraram menor preocupação a respeito de seu peso e forma corporal do que aqueles menos ativos. 
Ainda nesta temática, Dobmeyer et al. (2005) realizaram um estudo no qual investigaram a relação da preocupação com o ganho de peso e imagem corporal em militares que haviam parado de fumar. Foi encontrado que a insatisfação com o corpo após a cessação do ato de fumar, as preocupações relacionadas com o ganho de peso e a intenção de retomar o tabaco (se ocorresse ganho de peso) são constantes em militares que tentam parar de fumar. Em adição, foi apresentado que os militares que apresentaram insatisfação com as suas imagens corporais eram mais predispostos a retomarem o fumo.

Com relação ao grupo de transtornos alimentares, dois estudos foram realizados (MILLER, 2002; ANTCZAK; BRININGER, 2008). No primeiro deles, Miller (2000) realizou um estudo longitudinal durante o curso de uma academia militar, no qual avaliou a satisfação corporal em homens militares com antecedente para a sintomatologia de transtorno alimentar. $\bigcirc$ estudo indicou que a satisfação com a imagem corporal diminuiu com o passar dos anos letivos, enquanto a sintomatologia de transtornos alimentares aumentou.

Da mesma forma, Antczak e Brininger (2008) estudaram a incidência de três tipos de transtornos alimentares; anorexia nervosa, bulimia nervosa e transtorno alimentar sem outra especificação, diagnosticados em militares do Exército dos Estados Unidos. Os autores relataram que os casos de transtorno alimentar aumentaram significativamente em 2006, quando comparado com os dados de 1998, e atribuem este fato ao aumento de insatisfação com a imagem corporal dos militares.

Três estudos fazem parte do terceiro grupo e buscaram compreender a relação de militares com a insatisfação com algum aspecto específico da aparência (GUNDERSON, 1965; KLAGES, et al., 2007, LULÁCS; MURÁNYI; TÚRY, 2007). No primeiro, Gunderson (1965) investigou uma população de jovens militares da Marinha, na qual verificou a insatisfação com a altura, peso, nível intelectual, e buscou estabelecer a relação destes fatores com o rendimento dos jovens. Os maiores índices de insatisfação ocorreram no sobrepeso e pessoas com peso abaixo do ideal. Altura e peso não pareceram ter um efeito sobre o desempenho militar, exceto nos extremos. Pessoas muito baixas e com maior grau de sobrepeso expressaram maior nível de insatisfação e também tiveram baixos índices de desempenho.

Klages et al. (2007) investigaram a saúde bucal de jovens militares e a relação da percepção de oclusão dentária, com o impacto psicossocial da estética dentária e com a história de tratamento ortodôntico dos militares. Os autores chegaram à conclusão de que o significado subjetivo da aparência dentária relativo às experiências sociais e relações afetivas pode contribuir para a busca de saúde bucal, e que militares que associam afetos negativos com seus dentes podem evitar lembretes de sua aparência dentária, incluindo higiene oral e dentária. 
No mesmo ano, Lulács, Murányi e Túry (2007) compararam estudantes militares com universitários em geral, no que diz respeito ao ideal atlético como fator de risco em homens jovens, com relação à prática de musculação e insatisfação com o corpo, o que poderia servir como incentivador de dismorfias musculares. Os autores alegam que, ao contrário do ideal feminino magro, o corpo masculino ideal é baseado no ideal atlético. $O$ índice de massa corporal (IMC) dos militares apresentou-se levemente abaixo do IMC dos universitários em geral, a utilização de esteroides foi levemente maior entre os militares, assim como a prática de musculação apresentou-se significativamente maior neste grupo. As descobertas feitas pelos pesquisadores demonstraram que os estudantes militares pareceram ter menos insatisfação corporal e alguns fatores de proteção contra as características psicopatológicas dos transtornos de imagem corporal.

No quarto grupo, Weeks e Tsao (20 I 0) investigaram a sensação do "membro fantasma" em um militar, soldado, 26 anos de idade que, gravemente ferido em combate, perdeu as duas pernas na parte central do fêmur. Os autores atribuíram esta investigação ao fato de que inúmeros militares sofrem amputações em guerras e continuam a ter a sensação de que o membro amputado ainda faz parte do corpo, podendo sentir dor, coceiras, cãibras, entre outros, o chamado "membro fantasma". Estudos com membros fatasmas foram um dos pioneiros na área da imagem corporal, no final do século XVI, com Ambroise Paré.

Cash e Pruzinsky (1990) afirmam que a imagem corporal possui papel preponderante na maneira como interagimos com nosso mundo social e como nos percebemos e nos relacionamos com o próprio corpo. Neste contexto, a compreensão das particularidades de cada grupo de pessoas e sua relação com os componentes da imagem corporal torna-se relevante. Contudo, apenas nove artigos abordaram a imagem corporal de homens militares. Tal constatação expõe uma marcante limitação de pesquisas nesta área, o que limita a criação de medidas preventivas e terapêuticas para este grupo de pessoas, que poderiam ser possíveis se houvesse um maior aprofundamento dos estudos neste campo.

$O$ interesse de estudiosos sobre a imagem corporal de militares deve residir nas particularidades deste grupo de pessoas. A formação da "identidade militar" consiste no desenvolvimento de atributos, tais como cooperação, autoconfiança, persistência, iniciativa, coragem, responsabilidade, disciplina, equilíbrio emocional, entusiasmo profissional, entre outros, que são voltados para a aceitação de valores julgados necessários para que um indivíduo se adapte às exigências da vida militar, peculiares às situações de combate. Tais atributos são trabalhados durante todo o ano de instrução, por meio de situações criadas pelos instrutores. 
Outra forma de trabalhar os atributos militares dos incorporados às Forças Armadas é por meio de exemplos de conduta oferecidos pelos superiores hierárquicos. É exigido que o militar alcance elevados índices de conhecimento profissional, preparo físico, preparo mental, abnegação, vontade de lutar, sentimento de grupo, crença na profissão e paixão pelo que realiza, tendo em vista o atributo de seus superiores na ordem hierárquica estabelecida entre as patentes (cargos) da profissão.

Todo este processo possui um referencial externo ideal de atributos do corpo que sustentam a disciplina militar, tão necessária no desempenho das atividades profissionais destas pessoas. Este profissional deve estar preparado para missões específicas, tais como: defesa de seu país, garantia da lei e da ordem, missões de paz no exterior, apoio à população em calamidades públicas, entre outras, sendo fundamental um processo disciplinar sistemático e rigoroso (BRASIL, 20 I 0). No entanto, neste processo, cada militar possui sua história de vida, suas experiências prévias e sua identidade corporal, que devem ser consideradas no contexto das Instituições Militares. Os treinamentos devem ser desenvolvidos respeitando a singularidade de cada indivíduo, o que poderá motivá-lo a alcançar o máximo de suas capacidades. Ademais, nenhum treinamento terá resultados além do potencial de cada sujeito.

Os estudos sobre o impacto das atividades físicas e militares sobre a imagem corporal destes sujeitos podem possibilitar ajustamentos nos programas de treinamento das Instituições Militares, visando um desenvolvimento saudável da identidade corporal e militar deste grupo de pessoas. Com profissionais fortalecidos, há, consequentemente, um fortalecimento das Instituições Militares do Brasil. Diante destas questões, torna-se preocupante o resultado desta revisão de literatura, que revela que os estudos da imagem corporal de homens militares são escassos, não somente no Brasil, mas também no exterior.

\section{CONCLUSÃO}

objetivo deste trabalho foi realizar uma revisão de literatura dos estudos que versam sobre a imagem corporal de homens militares e verificar a relação desta com a prática de atividade física no contexto das Instituições Militares. Foi possível evidenciar a existência de uma acentuada lacuna nesta área, visto que apenas nove estudos foram encontrados abordando o tema. $\bigcirc$ tabagismo e a obesidade, os transtornos alimentares, a insatisfação com partes do corpo e o "membro fantasma" foram os temas encontrados.

Dos nove estudos encontrados, apenas dois estabeleceram algum tipo de relação entre a atividade física e a imagem corporal dos militares, demonstrando que este tema parece não estar sendo objeto de estudo dos pesquisadores, embora uma das características inerentes à profissão militar seja sua ligação com a atividade física. 
Considerando esta lacuna, sugerimos estudos futuros que investiguem as influências das atividades físicas e militares sobre as diferentes dimensões da imagem corporal de homens militares, a fim de propor medidas que potencializem os reflexos positivos e amenizem os impactos negativos das instruções e programas de atividades tipicamente militares no referido constructo.

\section{Body Image of the Military: a Review Study}

ABSTRACT: Body image is the mental representation of body identity, which makes its study important to the individualization of educational, pedagogical, and physical activities in different groups of individuals, including military ones. This study aimed to review the literature about the body image of military men and check its relationship with physical activity in the context of the military. Studies in the databases Scopus, SportDiscus, Web of Science, PsycINFO and Scielo have been tracked using search engine words related to body image, the military and physical activity. From a total of 10865 articles found, only nine were about body image among members of the military. There is a lack of studies in this area, which makes future research about body image of members of military institutions necessary, in order to benefit this group. KEYWORDS: Military; body image; body dissatisfaction; physical education.

\section{La imagen corporal de los militares: un estudio de revisión}

RESUMEN: La imagen corporal es la representación mental de la identidad corporal, lo que torna importante su estudio para la individualización de las acciones educativas, pedagógicas y de actividades fisicas en diferentes grupos de individuos, inclusive entre los militares. Este estudio tuvo como objetivo realizar una revisión de la literatura sobre la imagen corporal de hombres militares y comprobar la relación de esta con la práctica de actividad física en el contexto de las Instituciones Militares. Se buscaron estudios en las bases de datos Scopus, SportDiscus, Scielo, Web of Science y PsycINFO por medio de descriptores de búsqueda referentes a la imagen corporal, militar y actividad fisica. De un total de 10865 artículos encontrados sólo nueve abordaban el tema imagen corporal de hombres militares. Por tanto, hay una carencia de estudios en esta área, lo que torna necesaria la realización de investigaciones futuras sobre la imagen corporal de militares, con el propósito de traer beneficios para este grupo. PALABRAS CLAVE: Militares; imagen corporal; insatisfacción corporal; actividad fisica.

\section{REFERÊNCIAS}

ANTCZAKA, A.J.; BRININGERA, T. L. Diagnosed eating disorders in the U.S. military: A nine year review. Eating Disorders: the journal of treatment \& prevention, Philadelphia, v. I6, 
n. 5, p. 363-377, out./dez. 2008. Disponível em: < http://www.ncbi.nlm.nih.gov/pubmed/| $882136 \mid>$. Acesso em: 22 abr. 2013.

BRASIL. Estado-Maior do Exército. Sistema de Instrução Militar do Exército brasileiro (SIMEB). Brasília: EME, 2008.

BRASIL. Estado-Maior do Exército. Formação Básica do Combatente-PPB/2. Brasília: EME, 2010.

CAFRI, G; THOMPSON, J.K. Measuring male body image: a review of the current methodology. Psychology of Men and Masculinity, Washington, v. 5, n. I, p. 18-29, jan. 2004. Disponível em: <http://www.periodicos.capes.gov.br >. Acesso em: 22 abr. 2013.

CAMPANA, A. N. N. B; TAVARES, M. C. G. C. F. Avaliação da imagem corporal: instrumentos e diretrizes para pesquisa. São Paulo: Phorte, 2009.

CASH, T.; PRUZINSKY, T. Body images: development, deviance and change. New York: Guilford, 1990.

DOBMEYER, A. C. et al. Body image and tobacco cessation: relationships with weight concerns and intention to resume tobacco use. Body Image, New York, v. 2, n. 2, p. 187-192, jun. 2005. Disponível em: <http://www.ncbi.nlm.nih.gov/pubmed//8089/86>. Acesso em: $22 \mathrm{abr} .2013$.

GHIRALDELLI, J. P. Educação física progressista: a pedagogia crítico-social dos conteúdos e a educação física brasileira. São Paulo: Loyola, 1988.

GINIS, K. A. M. et al. Mind over muscle? Sex differences in the relationship between body image change and subjective and objective physical changes following a 12-week strength-training program. Body Image, New York, v. 2, n. 4, p. 363-372, dez. 2005. Disponível em: < http://www.ncbi.nlm.nih.gov/pubmed/I808920I >. Acesso em: 22 abr. 2013.

GINIS, K. A. M.; BASSETT, R. L. Exercise and changes in body image. In: CASH, T. F; SMOLAK, L. Body image: a handbook of science, practice, and prevention. Nova York: Guilford, 201 I, p. 378-386.

GRAMMAS, D. L; SCHWARTZ, J. P. Internalization of messages from society and perfectionism as predictors of male body image. Body Image, New York, v. 6, n. I, p. 31-36, jan. 2009. Disponível em: <http://www.ncbi.nlm.nih.gov/pubmed//9046933>. Acesso em: 22 abr. 2013.

GUNDERSON, E.K.E. Body size, self-evaluation, and military effectiveness. Journal of Personality and Social Psychology, Washington, v. 2, n. 6, p. 902-906, dez. 1965. Disponível em: < http://www.ncbi.nlm.nih.gov/pubmed/589|30। >. Acesso em: 22 abr. 2013. 
GUTIERREZ, W. A história da Educação Física. Porto Alegre: IPA, 1980.

JAMES, L. C. et al. The Tripler Army Medical Center LEAN Program: a healthy lifestyle model for the treatment of obesity. Military Medicine, Maryland, v. I62, n. 5, p. 328-332, mai/I997. Disponível em: < http://www.periodicos.capes.gov.br>. Acesso em: 22 apr. 2013.

KLAGES, $U$ et al. Perception of occlusion, psychological impact of dental esthetics, history of orthodontic treatment and their relation to oral health in naval recruits. Angle Orthodontist, v. 77, n. 4, p. 675-680, jul.2007. Disponível em: < http://www.ncbi.nlm.nih.gov/pubmed/I7605485>. Acesso em: 22 abr. 2013.

LULÁCS, L; MURÁNYI, I; TÚRY, F. Body dissatisfaction related to non-competitive bodybuilding in young males. Mentalhigiene es Pszichoszomatika, Budapeste, v. 8, p. I 3 | - | 45, feb. 2007. Disponível em: <http://www.periodicos.capes.gov.br>. Acesso em: 22 apr. 2013.

MCCREARY, D.R. Body image and muscularity. In: CASH, T. F.; SMOLAK, L. Body image: a handbook of science, practice, and prevention. New York: Guilford, 20 I I, p. I98-205.

MILLER, S. S. Body image disturbance in males: an antecedent to eating disorders. 2000. 238 f. Dissertação. Department of Graduate Psychology, Azusa Pacific University, Califórnia, 2000. Disponível em: <http://books.google.com.br/books?id=egEhtwAACAAJ >. Acesso em: 22 abr. 2013.

SABINO, C. Drogas de Apolo. In: GOLDENBERG, M. Nu e vestido. 2. ed. Rio de Janeiro, 2007, p. 139- 185.

SCHILDER, P. A imagem do corpo: as energias construtivas da psique. São Paulo: Martins Fontes, 1980.

SWAMI, V. et al. Personality and individual difference correlates of positive body image. Body image, New York, v. 5, n. 3, p. 322-325, 2008. Disponível em: < http://www.ncbi.nlm.nih. gov/pubmed/I8585107>. Acesso em: 22 abr. 2013.

TAVARES, M. C. G. C. F. Imagem corporal: conceito e desenvolvimento. Barueri: Manole, 2003.

TURTELLI, L.S.; TAVARES, M. C. G. C. F; DUARTE, E. Caminhos da pesquisa em imagem corporal na sua relação com o movimento. Revista Brasileira de Ciências do Esporte, Florianópolis, v. 24, n. I, p. 151-166, set. 2002. Disponível em: <http://rbceonline.org.br>. Acesso em: 22 abr. 2013.

WARD, K. D. et al. Characteristics of highly physically active smokers in a population of young adult military recruits. Addictive Behaviors, Philadelphia, v. 28, p. I 405- I 4 I 8, 2003. Disponível em: <http://www.ncbi.nlm.nih.gov/pubmed/I45 I2063>. Acesso em: 22 abr. 2013.

WEEKS, S. R.; TSAO, J. W. Incorporation of another person's limb into body image relieves phantom limb pain: a case study. Neurocase, Londres, v. I6, n. 6, p. 46। -465, abr. 20 I 0. Disponível em: <http://dx.doi.org/I0. I080//355479 I003730592 >. Acesso em: 22 abr. 20 I 3. 
Recebido em: 20 jul. 2010 Aprovado em: 27 ago. 201 I

Endereço para correspondência: Jairo José Monteiro Morgado Rua Umberto Nery número 95

Bairro Aeroporto Juiz de Fora-MG CEP:36038-290 